\title{
EDITORIAL
}

\section{EI tránsito a la autogestión hospitalaria en Chile}

Estamos frente al desafío de lograr que nuestros hospitales públicos se transformen, más que por el solo mandato de la ley, de estructuras rígidas a organizaciones dinámicas. Por cierto, existen avances significativos en esta materia, principalmente en aquellos establecimientos que iniciaron junto al proceso de reforma sectorial el camino a la autogestión hospitalaria. He aquí un primer punto.

La autogestión, de acuerdo a la RAE (Real Academia Española), se define como "el sistema de organización de una empresa según el cual los trabajadores participan en todas las decisiones". Para el caso de la organización llamada hospital, esta definición contiene elementos de política de recursos humanos que requieren de un transitar, un proceso, que de por sí es complejo. En efecto, los sistemas organizacionales en el ámbito sanitario se han preocupado históricamente solo de introducir cambios en el sistema de gestión, en el financiamiento o en la provisión de servicios, pero pocos han incorporado estrategias que apunten al corazón de las transformaciones organizacionales, es decir, a quienes efectivamente impulsan el cambio: el capital humano.

Para los equipos de trabajo en salud, es fundamental la presencia de una serie de estrategias que incluyan en detalle la implementación etapa a etapa, y los dejen en libertad de acción en pos del logro de los objetivos que se planteen. De ahí la importancia de fortalecer el proceso de toma de decisiones de los equipos directivos, en una primera etapa, para luego traspasar este fortalecimiento a los diferentes equipos de trabajo al interior de cada organización. Sin el desarrollo de competencias directivas que apunten al empoderamiento de todo el equipo y no solo del directivo superior, el sistema de alta dirección hospitalaria puede perder fuerza en su afán de convertir a los establecimientos de salud en organizaciones dinámicas e independientes.

Cabe preguntarnos si en Chile se han incluido las suficientes estrategias para la gestión del cambio al interior de nuestros hospitales, orientadas a la mejora de la educación y entrenamiento, a la reestructuración de las remuneraciones y a la implementación de incentivos al desempeño, propia de muchas organizaciones de servicios. Las políticas de recursos humanos del sector se han constituido en un aspecto central de la política sanitaria en nuestro país, lo que demuestra la importancia que tiene para los tomadores de decisión el rol del capital humano en la transformación hospitalaria. Para superar la constante rotación de directivos y la oposición per se a nuevos cambios que se quieran introducir, qué mejor que seguir ejecutando estrategias de cambio organizacional en conjunto con los propios equipos, pues así ellos lideran su propios planes de acción y adaptan las estrategias nacionales a su realidad local.

En este mismo sentido, contar con mejores equipos directivos implica también otorgar el más amplio espacio para la acción y toma de decisiones. El definir las atribuciones y el nivel de decisión resulta fundamental para la implementación de la estrategia, toda vez que trae más beneficios el incluir a los directivos locales en las decisiones propias de su organización, con amplia autonomía y voz propia. Para lograr esto, también resulta fundamental continuar con el proceso de adquirir y fortalecer competencias directivas, asegurando así la permanencia de directivos en etapas prolongadas del desarrollo de sus organizaciones. En Chile, particularmente con la implementación de la figura de los establecimientos de autogestión en red, definidos como centros sanitarios de alta complejidad que deben cumplir con procedimientos de medición de costos, de calidad de las atenciones prestadas y de la correcta satisfacción de sus usuarios y usuarias, se introduce autonomía en la selección e implementación de modelos de gestión que logren cumplir con estos procedimientos.

Para continuar con una implementación exitosa, se requiere continuar con la conceptualización de autogestión hospitalaria por parte de los equipos de salud. Esta constante conceptualización alimenta la discusión y, por cierto, la crítica interna, que permitirá el éxito de las estrategias 
definidas bajo los distintos modelos de gestión que implementen los Establecimientos de Autogestión en Red. Se debe considerar que una competencia abierta entre prestadores públicos debe y puede generar réplica de experiencias exitosas, creación de red de hospitales con colaboración recíproca, generación de polos de desarrollo; mirada así, la competencia es sana. En paralelo, esta práctica fuerza a cada hospital a definir su estructura de oferta (cartera de servicios) y tiende a priorizar la resolución de listas de espera históricas.

Finalmente, es necesario exponer que los esfuerzos realizados durante los últimos años tienen que ver con una política sanitaria integral y que se encuentra en etapa de proponer respecto de cómo evaluar el camino recorrido. El desafío ahora se traspasa desde la implementación de la política a la evaluación de ésta. Aquí se abren al menos dos nuevos desafíos: responder a las preguntas de los principales actores de la autogestión hospitalaria -los directivos- y a las de aquellos agentes que han acompañado de alguna u otra forma este proceso, es decir, las universidades.

La Escuela de Salud Pública de la Facultad de Medicina de la Universidad de Chile está realizando un aporte concreto en este ámbito. La institución cuenta con una experiencia pionera de acompañamiento a directivos de servicios de salud y de hospitales autogestionados en red, específicamente trabajando las competencias de los equipos directivos para el desarrollo de competencias esenciales en organizaciones públicas que prestan servicios de salud trabajando en red. Paralelamente, la realización del Programa de Actualización y Desarrollo para Ejecutivos en la Alta Dirección de Hospitales (PADE), es una contribución de alto nivel académico a la formación y capacitación de los equipos directivos.

El artículo de Vergara y Bisama, publicado en la presente edición de la Revista Chilena de Salud Pública, se refiere, precisamente, a las competencias esenciales que el mencionado programa de acompañamiento buscó desarrollar. Asimismo, en la sección "Cifras de Hoy" se entregan los datos hasta el momento disponibles, que permiten esbozar un panorama respecto de los resultados del proceso de autogestión hospitalaria. En el mismo contexto, el experto español Francesc Moreu nos da a conocer su visión de los logros y desafíos en materia de gestión hospitalaria, a partir de su vasta trayectoria internacional en el tema.
Alex Alarcón
Coordinador de Investigación
Escuela de Salud Pública 